\title{
EXTENDING FUNCTIONS TO OPERATE ON PROPER CLASSES
}

\author{
DAVID C. PETERSON
}

1. Introduction. The extension of addition of real numbers to addition of real-valued functions (done pointwise) is well known; as pointed out by A. P. Morse, it can be extended to (real-valued function)-valued functions, etc., as well. Morse gives a detailed description of set-theoretic machinery to accomplish this in $A$ theory of sets, the section "Direct Extensions." (All undetailed references to Morse herein are to this section.) To illustrate: If $g$ is the constant function of a real variable always returning a value of 1 , and $F$ is an appropriate extension of negation, then $F(g)$ is $-g$, the real-valued function always returning -1 .

However, if $g$ is not restricted to the real numbers, but is given the class of all sets as its domain, then $g$ is a proper class (not a set, i.e., not a member of any class) and $F(g)$ is undefined. This paper will investigate a canonical method of extending functions such as $F$ to operators capable of acting on proper classes. A necessary and sufficient condition for extendability is given and the dovetailing with direct extensions is discussed.

2. The basic extension. Consider a function such as negation defined for real-valued functions. If $f$ is included in $g$ then $-f$ is included in $-g$; more important,

$$
\text { if } g=\bigcup_{i \in I} f_{i}, \quad \text { then }-g=\bigcup_{i \in I}-f_{i} \text {. }
$$

Thus the image $-g$ of a function ${ }^{\prime \prime} g^{\prime}$ under negation is the union of ${ }^{\prime \prime}$ the images of its subsets. (Let $f$ range over all subsets of $g$.) This suggests that if negation, for example, has been defined only for real-valued functions that are sets, it would be appropriate to extend it to realvalued functions that are proper classes by means of a definition such as

$$
-G=\bigcup_{f \in \mathbf{s b} G}-f
$$

(where sb $G$ is the class of subsets of $G$ ). We would more generally define the extension of $F$, evaluated at $G$ to be $\bigcup_{f \in \mathrm{sb} G} F(f)$. This potential definition has three defects which fortunately are easily removed.

Received by the editors November 1, 1967. 
3. The final extension. First it is possible that not all of the subsets of $G$ are in the domain of $F$ (so that $F(f)$ in the union may not always be defined). Accordingly, we shall first modify the union as follows:

$$
\bigcup_{f \in \operatorname{sb} G \cap_{\text {dmn } F} F(f)}
$$

(where $\operatorname{dmn} F$ is the domain of $F$ ). Because of this modification, we will not accept the union as an appropriate value to be returned unless $\operatorname{sb} G \cap \operatorname{dmn} F$ is a nonempty cover of $G$, i.e.

$$
\operatorname{sb} G \cap \operatorname{dmn} F \neq \varnothing \quad \text { and } \quad U(\operatorname{sb} G \cap \operatorname{dmn} F)=G \text {. }
$$

In many applications, including the example of negation already discussed, there are many relations not functions which can be appropriately covered by functions and hence will have a negative. It is desirable that if the original function $F$ operates on function arguments only, then so should the extended operator. The second restriction accomplishes this in a natural manner by requiring that a nested cover of $G$ be a subclass of the cover sb $G \cap \operatorname{dmn} F$. Since the union of a nest of functions is a function, no relation not a function can be so covered by functions.

The final restriction is to simply specify an arbitrary class $A$ (not necessarily a proper class) to be excluded from consideration. We extend in the above manner the restriction of $F$ to $\sim A$. The usefulness of this restriction will become apparent in the final section of this paper where we discuss the dovetailing of this extension with Morse's direct extension. In other cases one may take $A=\varnothing$ if appropriate.

Definition 1. $G$ is appropriate for the extension of $F$ beyond $A$ means $G$ can be covered by a nonempty nested subclass of $\mathrm{sb} G$ $\cap \operatorname{dmn} F \cap \sim A$ and $G \notin A$.

Definition 2. Appro $F A$ is

$\{g: g$ is appropriate for the extension of $F$ beyond $A\}$.

Definition 3. The extension of $F$ beyond $A$, evaluated at $G$ is

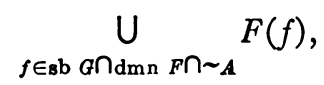

if $G$ is appropriate for the extension of $F$ beyond $A ; F(G)$, otherwise.

\section{Extendable functions.}

Definition $4 . F$ is extendable beyond $A$ means whenever both $F(G)$ and the extension of $F$ beyond $A$, evaluated at $G$ exist, they are equal. 
In this case the extension will only add new values, not change old ones. A simple necessary and sufficient condition for extendability is found in

Theorem 1. If $F$ is a function then $F$ is extendable beyond $A$ if and only if the restriction of $F$ to Appro $F A$ is monotonic (weakly increasing with respect to inclusion).

Proof. First suppose $F$ is extendable beyond $A, g$ and $h$ are members of dmn $F \cap A$ ppro $F \cup A$, and $g \subset h$. Then because $g \in \mathrm{sb} h$ רdmn $F \cap \sim A$,

$$
\begin{aligned}
F(g) & \subset \underset{f \in \operatorname{sb}}{\bigcup_{h \cap_{\mathrm{dmn}} F \cap \sim A}} F(f) \\
& =\text { the extension of } F \text { beyond } A \text {, evaluated at } h \\
& =F(h) .
\end{aligned}
$$

The arbitrary nature of $g$ and $h$ assure that the restriction of $F$ to Appro $F A$ is monotonic as desired. Next suppose the restriction of $F$ to Appro $F A$ is monotonic and $g \in \operatorname{dmn} F \cap A$ ppro $F A$. Then

$$
g \in \operatorname{sb} g \cap \operatorname{dmn} F \cap \sim A .
$$

Furthermore, whenever $f \in \operatorname{sb} g \cap \operatorname{dmn} F \cap \sim A$

$$
f \in \operatorname{sb} f \cap \operatorname{dmn} F \cap \sim A,
$$

thus $\{f\}$ is an appropriate cover to insure that $f \in$ Appro $F A$, and hence $F(f) \subset F(g)$. Thus

$$
\begin{aligned}
& F(g) \subset \bigcup_{f \in \mathrm{sb}_{0} \cap \mathrm{dmn} F \cap \sim \Delta} F(f) \\
& \subset \bigcup_{f \in \text { sb } g \cap \operatorname{dmn} F \cap \sim A} F(g) \\
& =F(g) \text {. } \\
& F(g)=\bigcup_{f \in \operatorname{sb} g \cap \operatorname{dmn} F \cap \sim A} F(f) \\
& =\text { the extension of } F \text { beyond } A \text {, evaluated at } g \text {. }
\end{aligned}
$$

The arbitrary nature of $g$ assures that $F$ is extendable beyond $A$ as desired and the proof is complete.

5. Extending direct extensions. The reader desiring to verify the proofs of results given in this section is referred to Morse for appropriate definitions and preceding theorems.

The extendability of Morse's direct extension is demonstrated in 
THEOREM 2. If $f$ is a function, $\operatorname{dmn} f \subset A$, and $\theta$ is monotonic, then the direct extension of $f$ beyond $A$ via $\theta$ is extendable beyond $A$.

This can be readily seen by verifying that the restriction of the direct extension to $\sim A$ is monotonic and applying Theorem 1.

When Morse's direct extension is applied to a function $f$ of one variable, $\theta$ is generally selected to be an identity function with appropriate domain. In this case, $\theta$ is inherently monotonic and Theorem 2 applies. Difficulties can be encountered, however, when a function of two variables is extended. In this case, $\theta$ must be a function of two function variables which returns as its value a function which in turn returns ordered pairs:

$$
\theta(f, g)(t)=(f(t), g(t)) .
$$

It can easily be checked that such $\mathrm{a} \theta$ is monotonic if pairing is also:

$$
\left(f_{0}, g_{0}\right) \subset\left(f_{1}, g_{1}\right) \quad \text { whenever } f_{0} \subset f_{1} \text { and } g_{0} \subset g_{1} \text {. }
$$

An even stronger property of ordered pairs is required to insure that an ordered pair of functions can be covered by a nest of ordered pairs of functions. Ideally one would hope that

$$
\bigcup_{i \in I}\left(f_{i}, g_{i}\right)=\left(\bigcup_{i \in I} f_{i}, \bigcup_{i \in I} g_{i}\right) \text {. }
$$

The commonly used pair of Weiner, besides not being properly defined for classes, fails in both these respects. The pair of J. W. Weihe fails in some instances, whereas the modification found in Morse, the section "Ordered Pairs," succeeds completely. (Weihe's pair $(F, G)$ is the cartesian product of sb $F$ and $\operatorname{sb} G$ formed using Weiner's pairs. The successful pair is formed using the classes of subsets of cardinality $\leqq 1$ in place of $\operatorname{sb} F$ and sb $G$ in Weihe's pair; other modifications in Morse are irrelevant here.)

\section{REFERENCE}

1. A. P. Morse, $A$ theory of sets, Academic Press, New York, 1965.

United States Air Force Academy 